\title{
Dismorfia Muscular e o Uso de Suplementos Ergogênicos em Desportistas
}

\author{
Muscle Dysmorphia and the Use of Ergogenic \\ Supplements in Athletes
}

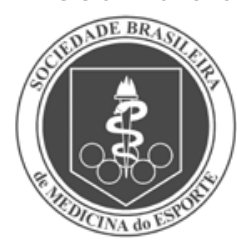

Artigo Original

Litiane Dorneles de Lima' ${ }^{1}$

Cristina Machado Bragança de Moraes² Vanessa Ramos Kirsten ${ }^{3}$

1. Graduada em Nutrição pelo Centro Universitário Franciscano (UNIFRA) de Santa Maria, RS.

2. Graduada em Nutrição pela UNIJUÍRS; Professora do Curso de Nutrição e Pós-Graduação do Centro Universitário Franciscano (UNIFRA) de Santa Maria, RS; Mestre em Ciência da Nutrição Unicamp - Campinas, SP; Doutoranda em Biologia Celular e Molecular - PUC - Porto Alegre, RS.

3. Graduada em Nutrição pela UNIFRARS; Professora do Curso de Nutrição e Pós-Graduação do Centro Universitário Franciscano (UNIFRA) de Santa Maria, RS; Mestre em Ciência da Saúde PUCRS; Doutoranda em Saúde da Criança e do Adolescente - UFRGS.

Endereço para correspondência: Cristina Machado Bragança de Moraes Rua Tuiuti, 1.336, apto. 201 - Centro 97015-662 - Santa Maria, RS E-mails: cbmoraes@unifra.br, c_bmoraes@yahoo.com.br

\section{RESUMO}

A dismorfia muscular (DM) é um transtorno da imagem corporal que acomete principalmente homens que, apesar da grande hipertrofia muscular, consideram-se pequenos e fracos. Tendo em vista o crescente uso de substâncias ergogênicas para o aumento de massa muscular e o elevado número de indivíduos com transtornos corporais, o objetivo deste trabalho foi avaliar a presença de DM e o uso de suplementos ergogênicos em desportistas frequentadores de academia. Foram avaliados 23 indivíduos do sexo masculino com média de idade de $24 \pm 3,8$ anos, praticantes de treinamento de força, com idades entre 18 e 35 anos. Para a avaliação corporal foi realizado o teste de bioimpedância, com o qual se obteve os dados de massa magra e gorda, respectivamente, de 66,5 $\pm 10,1 \mathrm{~kg}$ e 10,5 $\pm 3,1 \mathrm{~kg}$. Para detectar o risco de DM, foi usada uma escala (MASS - Muscle Appearance Satisfaction Scale); se o resultado desta fosse acima de 52 pontos, estes apresentavam o risco de DM. Juntamente, foi respondido outro questionário para o conhecimento do uso de suplementos. A análise dos resultados identificou que, dos avaliados, 17,4\% $(n=4)$ apresentaram risco positivo para o desenvolvimento deste transtorno corporal; porém, destes, apenas $25 \%$ declararam fazer uso de suplementos alimentares. Conclui-se que não houve relação entre o uso de suplementos e a presença de dismorfia muscular.

Palavras-chave: suplementos alimentares, avaliação nutricional, imagem corporal.

\begin{abstract}
Muscle Dysmorphia (MD) is a body image disorder that affects especially men, who despite the large muscular hypertrophy, consider themselves tiny and weak. Considering the increasing use of ergogenic substances to increase muscular mass and the large number of individuals with body disorders, this research aim was to evaluate the presence of MD and the use of ergogenic supplements by athletes who go to health clubs. The study sample was formed by 23 male individuals with mean age of $24 \pm 3.8$ years, who practice strength exercises and and aged between 18 and 35 years. Body was assessed through a bioimpedance test from which it was possible to get the data of lean and fat mass, which were $66.5 \pm 10.1 \mathrm{Kg}$ and $10.5 \pm 3.1 \mathrm{Kg}$, respectively. DM risk was detected with a scale (MASS- Muscle appearance satisfaction scale). If the result was above 52 points, it presented DM risk. Additionally, a questionnaire was answered to provide information about the use of supplements. The results analysis showed that among the evaluated sample, 17.4\% ( $n=4)$ demonstrated positive risk to the development of body disorders, but only $25 \%$ of them declared the use of food supplements. It was concluded that there was not correlation between use of supplements and presence of muscle dysmorphia.
\end{abstract}

Keywords: food supplements, nutritional evaluation, body image.

\section{INTRODUÇÃO}

Há uma grande pressão da mídia e da sociedade em relação ao melhoramento corporal, o que contribui para maior procura por academias, assim como pelo uso de suplementos e anabolizantes ${ }^{(1)}$; porém, o excesso de atividade física pode estar relacionado com riscos à saúde, entre eles os transtornos de imagem corporal, como a dismorfia muscular (DM) (2).

A busca por um corpo esteticamente perfeito e a falta de uma cultura corporal saudável tem levado a população a usar substâncias que possam potencializar, no menor espaço de tempo possível, os seus objetivos ${ }^{(3)}$.
Buscando uma melhor performance, atletas e desportistas em geral recorrem ao uso de recursos ergogênicos. Estes podem ser nutricionais, físicos, mecânicos, psicológicos, fisiológicos ou farmacológicos. São tratamentos ou substâncias elaborados para aumentar o desempenho desportivo ou aprimorar a capacidade de realizar um trabalho físico ${ }^{(4)}$.

A academia é um local para a prática de exercícios físicos por indivíduos sem vínculos profissionais com o esporte. Este ambiente favorece a disseminacão de padrões estéticos estereotipados, como o corpo com baixa quantidade de gordura e com elevado volume de tônus muscular ${ }^{(5)}$. 
Preocupações mórbidas com a imagem corporal foram consideradas exclusivas do gênero feminino, até pouco tempo ${ }^{(2)}$, associadas aos quadros de anorexia reversa e bulimia nervosa ${ }^{(6)}$. Mais recentemente, estas preocupações também têm sido encontradas em homens, definida inicialmente como anorexia nervosa reversa e posteriormente renomeada dismorfia muscular $(\mathrm{DM})^{(7)}$.

A dismorfia muscular é caracterizada como um transtorno da imagem corporal apresentada por atletas e/ou desportistas e foi descrita por Pope et al. em 1993(8), os quais inicialmente denominaram a dismorfia muscular como "anorexia nervosa reversa". Posteriormente, Pope et al., em 1997(7), verificaram que os atletas não apresentavam disfunções no âmbito nutricional e sim distorções na imagem de sua composição muscular, modificando a denominação para dismorfia muscular (DM).

A DM envolve uma preocupação por parte dos indivíduos praticantes de exercícios de força de não serem suficientemente fortes e musculosos ${ }^{(2)}$.

Os indivíduos acometidos por este transtorno frequentemente se descrevem como "fracos e pequenos", quando, na verdade, apresentam musculatura desenvolvida em níveis acima da média da população masculina, caracterizando uma distorção da imagem corporal(9).

Tendo em vista o aumento de publicações relatando a presença desta dismorfia, o objetivo deste trabalho foi verificar a prevalência de DM em desportistas de academias de uma cidade do interior do Rio Grande do Sul (RS) e verificar se existe relação com o uso de suplementos ergogênicos.

\section{MÉTODOS}

\section{Amostra}

A amostra foi constituída por indivíduos do gênero masculino, aleatoriamente selecionados em academias do centro da cidade de Santa Maria/RS, entre agosto e novembro de 2008.

Antes de qualquer intervenção, os participantes assinaram o TCLETermo de Consentimento Livre e Esclarecido.

Como critérios de inclusão foram considerados que os indivíduos fossem declaradamente saudáveis, ter idade entre 18 e 35 anos de idade, com prática de exercícios de hipertrofia muscular regular.

Este trabalho foi aprovado pelo Comitê de Ética e Pesquisa com Seres Humanos do Centro Universitário Franciscano (UNIFRA) sob o número de protocolo 163.2009.2.

Para a avaliação do risco da dismorfia muscular, foi aplicada uma escala denominada Muscle Appearance Satisfaction Scale (MASS), um instrumento de satisfação da aparência muscular, validado e traduzido para o português como escala de satisfação da aparência muscular. Este instrumento apresenta 19 itens relativos a autopercepção corporal e hábitos relacionados à prática de exercícios de fortalecimento muscular, objetivando obter subsídios para identificar a DM. Esse instrumento tem sido reconhecido e utilizado pela literatura psiquiátrica para auxiliar no diagnóstico de $\mathrm{DM}^{(9)}$. O MASS fornece um somatório de todos os itens (LMASS). Segundo o pesquisador que desenvolveu a escala, escores globais superiores a 52 pontos no MASS parecem ser indicativos de $\mathrm{DM}^{(10)}$. A versão em português foi considerada como uma reprodução fiel e apropriada por dois especialistas ${ }^{(11)}$ que avaliaram tanto o idioma quanto o conteúdo da tradução.

Concomitantemente, foi entregue um questionário não validado de autopreenchimento com questões fechadas referente ao uso de suplementos ergogênicos.

A coleta dos dados antropométricos foi de acordo com o protocolo de Petroski ${ }^{(12)}$, e realizada pela própria pesquisadora. Foi aferido o peso corporal utilizando-se uma balança digital do tipo portátil devidamente testada e calibrada da marca GAMA Professional (KH-2103), com capacidade máxima de $150 \mathrm{~kg}$ e precisão de $100 \mathrm{~g}$. O indivíduo posicionou-se em pé, no centro da base da balança, com os braços estendidos ao longo do corpo e descalço. Para obter-se a altura do sujeito, utilizou-se o estadiômetro compacto tipo trena (Sanny ${ }^{\circledR}$ ES-2040), no qual o indivíduo ficou em pé, ereto, descalço, com os calcanhares juntos, costas retas, ombros e glúteos em contato com o estadiômetro, os braços estendidos ao lado do corpo e com a cabeça erguida olhando para um ponto fixo na altura dos olhos.

Com a obtenção dos dados de peso e altura foi realizada a avaliação corporal através do método da bioimpedância (BIA), em que o voluntário teve que seguir algumas recomendações como: (a) não comer ou beber a menos de quatro horas antes do teste; (b) não fazer exercícios a menos de 12 horas antes do teste; (c) urinar 30 minutos antes do teste; (d) não consumir álcool a menos de 48 horas do teste; (e) não tomar medicamentos diuréticos a menos de sete dias do teste; (f) permanecer, pelo menos, cinco minutos deitado em decúbito dorsal, em total repouso antes da execução do teste ${ }^{(13)}$.

A bioimpedância foi realizada antes do início do treino, com o indivíduo em decúbito dorsal, com braços e pernas confortavelmente separados, em uma superfície isolante (maca), sem portar qualquer objeto metálico, com colocação de eletrodos em quatro pontos estratégicos (mão, pulso, pé e tornozelo). O aparelho usado foi de disposição tetrapolar, da marca Biodinamics A- 310. Após a passagem da corrente elétrica pelo corpo do atleta, o instrumento forneceu dados de porcentagem de gordura corporal (MG), massa magra (MM), resistência, reactância e taxa metabólica basal (TMB).

Os dados obtidos foram analisados através de estatística descritiva simples (média, desvio padrão e porcentagens). Para comparação entre média foi usado o teste $t$ de Student, e para a comparação de variáveis categóricas usou-se o teste do Qui-quadrado. O software usado foi o SPSS 15.0 e foram consideradas diferenças estatísticas quando $p<0,05$. O teste de Fisher foi utilizado para verificar as possíveis associações e avaliar as diferenças significativas entre as variáveis categóricas.

\section{RESULTADOS}

Participaram do estudo 23 voluntários do gênero masculino, com média de idade de $24 \pm 3,8$ anos, peso médio de 75,9 \pm 9,4kg, 66,5 \pm $10,1 \mathrm{~kg}$ de massa magra (MM) e 10,5 $\pm 3,1 \mathrm{~kg}$ de massa gorda (MG).

Conforme a tabela 1, com relação ao tempo (meses) de atividade física, pode-se observar que, da amostra avaliada, 47,8\% ( $n=11)$ praticam academia há mais que seis meses, assim como 73,9\% ( $n=17)$ dos indivíduos têm o objetivo de ganho de MM.

A maioria dos participantes, $65,3 \%(n=15)$ têm uma frequência de exercícios de três a cinco vezes semanais e, em relação ao uso de substâncias ergogênicas, 26,1\% $(n=6)$ referem usar algum tipo de suplementação (tabela 1) e, destes, 73,9\% ( $n=17$ ), o fazem sem prescrição. Os demais indivíduos que relatam fazer uso de suplementos sem prescrição informaram que seguem a indicação de amigos, $13 \%(n=3)$, a indicação de personal trainner, lojas de suplementos e nutricionistas 4,3\% ( $n=1)$.

Ao se relacionar a composição corporal entre indivíduos com risco e sem risco de DM (tabela 2), observou-se que não houve diferença significativa entre a composição corporal, ou seja, MM e MG; porém, indivíduos que mostraram risco positivo de dismorfia muscular apresentaram menor média de massa gorda (MG). Este resultado pode ser devido à grande preocupação com a prática regrada de exercícios físicos e também com sua alimentação.

Observando a tabela 2, nota-se uma diferença em relação ao uso e ao não uso de suplementos ergogênicos por parte destes indivíduos, 
Tabela 1. Descrição de dados de indivíduos praticantes de exercício de força de academias de Santa Maria, RS.

\begin{tabular}{l|c|c}
\hline Variáveis & $\%$ & $\mathbf{n}$ \\
\hline TEMPO & & \\
\hline$>6$ meses & 47,8 & 11 \\
\hline $3-6$ meses & 17,4 & 4 \\
\hline$<3$ meses & 34,8 & 8 \\
\hline OBJETIVO & & \\
\hline Ganhar MM & 73,9 & 17 \\
\hline Manutenção de PC & 14,4 & 4 \\
\hline Perda de PC & 8,7 & 2 \\
\hline FREQUÊNCIA DE TREINO SEMANAL & & 15 \\
\hline 3-5 & 65,3 & \\
\hline$>5$ & 34,8 & 6 \\
\hline USO DE SUPLEMENTOS & & \\
\hline Sim & 26,1 & \\
\hline Não & 73,9 & \\
\hline
\end{tabular}

$\mathrm{MM}=$ massa magra; $\mathrm{PC}=$ peso corpóreo.

Tabela 2. Consumo de suplementos, MM, MG e risco de DM em indivíduos praticantes de exercícios de força em academias de Santa Maria, RS.

\begin{tabular}{l|c|c|c}
\hline & Sem risco de DM & Com risco de DM & \\
\hline & $\%(\mathrm{n})$ & $\%(\mathrm{n})$ & \\
\hline Consomem suplemento & $26,3(5)$ & $25(1)$ & \\
\hline Não consomem suplemento & $73,7(14)$ & $75(3)$ & \\
\hline & Média $\pm \mathrm{DP}$ & Média $\pm \mathrm{DP}$ & $\mathrm{P}$ \\
\hline MM $(\mathrm{kg})$ & $65,4 \pm 7,5$ & $65,5 \pm 6,1$ & 0,981 \\
\hline MG (Kg) & $10,6 \pm 3,1$ & $8,5 \pm 1,9$ & 0,219 \\
\hline $\mathrm{P}=0,730(\mathrm{p}$ corrigido pelo teste de Fisher) $n=$ número de individuos $\%$ DM $=$ dismorfia muscular
\end{tabular}

verificando-se que, dos indivíduos que apresentaram risco positivo de DM, apenas um (25\%) relatou o consumo de suplementos.

Com relação à prevalência de risco de DM, identificou-se nos avaliados $17,4 \%(n=4)$ de risco positivo para o desenvolvimento deste transtorno corporal (figura 1).

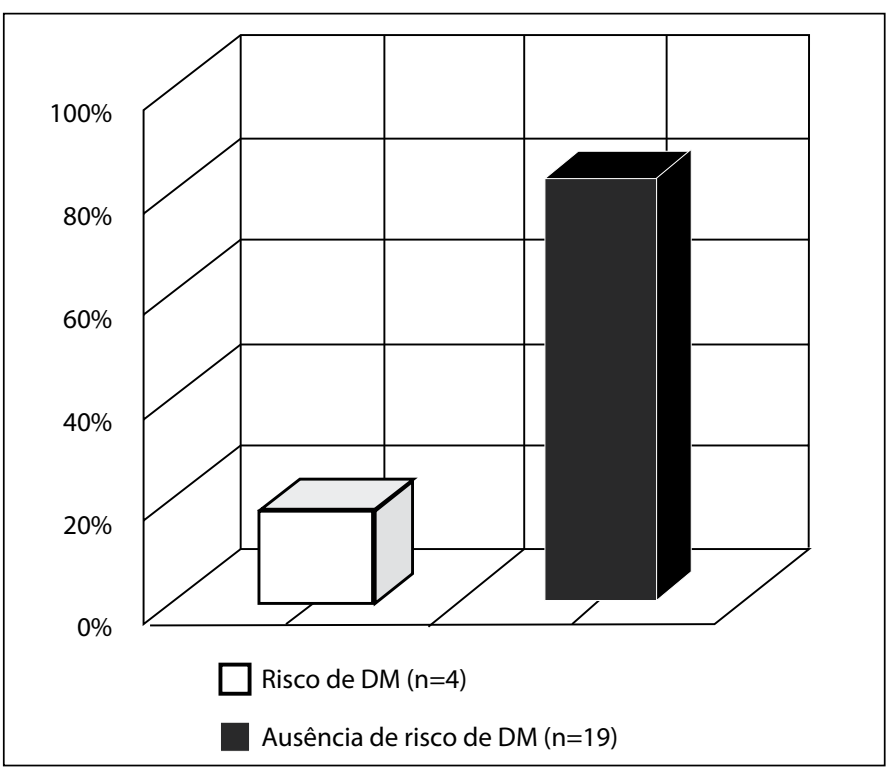

Figura 1. Prevalência de dismorfia muscular em indivíduos praticantes de exercícios de força em academias de Santa Maria, RS.

\section{DISCUSSÃO}

A descrição inicial da DM deu-se por Pope et al., em 1993(8), em uma amostra de indivíduos levantadores de peso na qual evidenciouse uma correlação positiva entre o uso de esteroides anabolizantes e a presença do transtorno. Observaram, ainda, que quatro entre nove indivíduos que apresentaram DM desenvolveram um quadro do transtorno corporal após fazer uso de suplementos ergogênicos, sugerindo que estes podem ter relação ou ser um fator de risco na percepção da imagem corporal.

Referindo-se à dismorfia muscular, neste estudo, verificou-se que, dos quatro indivíduos que apresentavam os sinais de DM, um deles fazia uso de suplemento.

Pope et al., em estudo realizado no ano de $1997^{(7)}$ com 16 indivíduos, descreveram prevalência de 10\% de DM entre levantadores de peso e de até $84 \%$ entre fisiculturistas que participavam de competições; destes, foi percebido o uso de esteroides anabolizantes em $100 \%$ dos homens.

Olivardia et al., em 2000(14), verificaram que indivíduos frequentadores de academias de ginástica que faziam levantamento de peso e apresentavam DM tinham maior prevalência de uso de suplementos do que os indivíduos nas mesmas condições sem o transtorno.

Verificou-se também, nesta pesquisa, que, dentre os indivíduos sem risco de DM ( $n=19)$, cinco $(26,3 \%)$ consumiam suplementos ergogênicos, os demais negaram o uso de qualquer substância ergogênica.

Corroborando os dados do estudo de Miarka et al. (2007) $)^{(15)}$ que avaliou o uso de suplementos alimentares em 247 estudantes da área de educação física e, destes, 26,64\% faziam uso de substâncias ergogênicas, restando $73,36 \%$ dos estudantes que não realizavam o uso. Dados parecidos foram encontrados no estudo de Schneider e Machado (2006) $)^{(16)}$, em pesquisa realizada na cidade de Porto Alegre (RS), com frequentadores de academias de ginástica, que analisou 65 participantes, observou que $24(36,9 \%)$ consomem algum tipo de suplemento alimentar e $41(63,1 \%)$ não consomem estes produtos; destes, 54,54\% eram do sexo masculino.

Sabe-se que alguns suplementos apresentam efeitos comprovados no aumento da MM; porém, os estudos, na maioria deles, são controversos e pouco produtivos.

No presente estudo observou-se o uso de suplementos sem prescrição, contrariando os dados de Araujo et al. (2002)(17), em estudo realizado em academias cadastradas na Federação de Fisiculturismo em Goiânia, mostrou que, na maioria da sua amostra, houve o relato do uso de suplementos com a indicação de nutricionista e/ou professores/ instrutores da academia. Em outro estudo, realizado por Miarka et al. (2007) (15), com estudantes de educação física, verificou-se que, quando o suplemento era consumido por indicação, a principal fonte de prescrição era o instrutor, seguido de treinador, amigos, vendedor de loja e, por último, o nutricionista; dados estes diferentes das informações encontrados nesta pesquisa, sendo que é competência do nutricionista a prescrição de suplementos alimentares.

Ao avaliar a distribuição corporal, pode-se perceber que, mesmo sem diferença significativa entre os indivíduos com e sem risco para DM, a MG foi menor nos indivíduos com DM, dado semelhante ao apresentado por Olivardia et al. (2000)(14), em estudo realizado em Boston com 54 pessoas fisicamente ativas, das quais $44 \%$ apresentavam risco de DM e uma quantidade de massa gorda inferior aos indivíduos que não apresentaram risco de DM.

Ao final deste estudo, pode-se concluir que a prevalência de risco de dismorfia muscular entre os indivíduos foi baixa, e que estes, apenas uma minoria, fazia uso de suplementos, embora se pôde perceber o receio por parte dos indivíduos em declarar o uso e substâncias licitas 
e ilícitas, assim como a exposição da composição corporal.

Este trabalho apresentou um número restrito de participantes, devido à resistência dos indivíduos em participarem do estudo por terem receio de declarar o uso de substâncias licitas ou ilícitas, assim como a exposição de sua composição corporal, em que pode-se observar uma grande negação dos indivíduos visualmente dismórficos.

É importante destacar a escassez de estudos com este tipo de transtorno dismórfico corporal que permitam comparações com os resultados deste estudo. Isso sugere a necessidade de ampliar os estudos sobre este tema, a fim de identificar e compreender a dismorfia muscular.

Todos os autores declararam não haver qualquer potencial conflito de interesses referente a este artigo.

\section{REFERÊNCIAS}

1. Stricker PR. Sports training issues for the pediatric athlete. Petriatr Clin N Am. 2002;49:793-802.

2. Assunção SSM. Dismorfia muscular. Rev Bras Psiquiatria. 2002;24:80-4.

3. Santos MAA, Santos RP. Uso de suplementos alimentares como forma de melhorar a performance nos programas de atividade física em academias de ginástica. Rev Paul Educ Fís. 2002;16:174-85.

4. Fontana KE, Valdes H, Baldissera V. Glutamina como suplemento ergogênico. Rev Bras Ciênc e Mov. 2003;11:91-6.

5. Saba FKF. Determinantes da prática de exercício físico em academias de ginástica [Tese]. São Paulo: Universidade de São Paulo, 1999.

6. Mehler PS. Bulimia nervosa. N Engl J Med. 2003;349:875-81.

7. pope HGJ, Gruber AJ, Choi P, Olivardia R, Phillips KA. Muscle Dysmorphia. An Underrrecognized Form of Body Dysmorphic Disorder. Psychosomatics. 1997;38:548-56.

8. Pope HGJ, Kartz DL, Hudson J. Anorexia nervosa and "reverse anorexia" among 108 male bodybuilders. Comp Psychiatry. 1993;34:406-9.

9. Cafri G, Van Den Berg P, Thompson JK. Pursuit of muscularity in adolescent boys: relations among biopsychosocial variables and clinical outcomes. J Clin Child Adolesc Psychol. 2006;35:283-91.
10. Mayville SB, Williamson DA, White MA, Netemeyer RG, Drab DL. Development of the Muscle Appearance Satisfaction Scale: A self - Report measure of the assessment of muscle dysmorphya symptons. Assesm. 2002;9:351-60.

11. Junior SHAS, Souza MA, Silva JHA. Tradução, adaptação e validação da escala de satisfação com a aparência muscular (MASS). Rev. Digital. Buenos Aires, maio, 2008. Disponível em: <http://www. efdeportes.com/>. v. 13, n. 120. Acesso em: 08 março. 2009.

12. Petroski EL. Antropometria: técnicas e padronizaçōes. Porto Alegre: Palloti, 1999

13. Heyward VL, Stolarczyk LM. Avaliação da composição corporal aplicada. 1. ed. São Paulo: Manole, 2000.

14. Olivardia R, Pope HGJ, Hudson Jl. Muscle Dysmorphia in Male Weghtlifters: A Case Control Study. Am J Psychiatry. 2000;157:1291-6.

15. Miarka B, Junior CCL, Interdonato GC, Vecchio FBD. Características da suplementação alimentar por amostra representativa de acadêmicos da área de educação física. Mov \& Percep. 2007;8:278-88.

16. Schneider AP, Machado DZ. Consumo de Suplementos Alimentares entre Frequentadores de uma Academia de Ginástica de Porto Alegre - Rio Grande do Sul. Rev Nut em Pauta. 2006;78:16-20.

17. Araujo LR, Andreolo J, Silva MS. Utilização de suplemento alimentar e anabolizante por praticantes de musculação nas academias de Goiânia - GO. Rev Bras Ciên e Mov. 2002;10:13-8. 